\title{
Diagnóstico preliminar de la molestia sanitaria causada por Culicoides (Diptera: Ceratopogonidae) en el departamento de Boyacá, Colombia
}

\author{
Erika Santamaría1, Olga Lucía Cabrera1', Yaneth Zipa², Cristina Ferro', \\ Martha Liliana Ahumada1', Raúl Hernando Pardo ${ }^{1,3}$ \\ ${ }^{1}$ Grupo de Entomología, Instituto Nacional de Salud, Bogotá, D.C., Colombia \\ ${ }^{2}$ Grupo de Enfermedades Transmitidas por Vectores, Secretaría de Salud de Boyacá, Tunja, Colombia \\ ${ }^{3}$ Grupo de Investigaciones en Entomología, Biología Celular y Genética, Departamento de Ciencias Básicas, \\ Universidad de La Salle, Bogotá D.C., Colombia \\ Lugar donde se realizó el trabajo: \\ Laboratorio de Entomología, Instituto Nacional de Salud, Bogotá, D.C., Colombia. \\ Catorce municipios del departamento de Boyacá, Colombia.
}

Introducción. Los habitantes del occidente del departamento de Boyacá han reportado molestia sanitaria y problemas dermatológicos ocasionados por la constante picadura de insectos del género Culicoides.

Objetivo. Identificar el área de Boyacá afectada por Culicoides, determinar las especies antropofílicas involucradas y su abundancia.

Materiales y métodos. Se solicitó información sobre la molestia sanitaria y el registro de casos dermatológicos asociados a la picadura de Culicoides a las autoridades de salud de los municipios que por sus reportes previos o por sus características geográficas se consideraron como potencialmente afectados. En los municipios que informaron sufrir la problemática, se realizó un muestreo entomológico con atrayente humano afuera de las viviendas.

Resultados. Se confirmó la gravedad de la molestia en el área rural de nueve municipios ubicados en el flanco occidental de la Cordillera Oriental. Aunque los registros epidemiológicos fueron fragmentados, se estableció que en seis municipios el $11,4 \%$ de los casos $(n=2.472)$ de dermatitis reportados entre el 2003 y el 2005 fueron atribuidos a la picadura de Culicoides. Los resultados entomológicos mostraron que la especie dominante fue Culicoides pachymerus, $99,3 \%$ de las 3.389 hembras recolectadas, con tasas de picadura (promedios geométricos) por municipio de hasta 52 hembras/persona en 5 minutos. Mediante análisis multivariado, se encontró que la abundancia de esta especie se relaciona negativamente con la altitud.

Conclusiones. Por su dominancia y altas tasas de picadura, $C$. pachymerus es muy probablemente la especie responsable de la molestia sanitaria y los problemas dermatológicos causados por Culicoides en el departamento de Boyacá.

Palabras clave: Ceratopogonidae, dermatitis, salud pública, ecosistema andino.

Preliminary evaluation of the Culicoides biting nuisance (Diptera: Ceratopogonidae) in the province of Boyacá, Colombia

Introduction. Inhabitants in the western border of Boyacá province have reported high nuisance levels and dermatologic problems caused by the intensely irritating bites of the very small flies of the genus Culicoides.

Objective. A survey was carried out to locate the affected area, identify the anthropophylic Culicoides species and estimate its abundance in Boyacá.

Materials and methods. Nuisance reports and clinical records of dermatologic cases associated with Culicoides bites were requested from health authorities in counties where nuisance reports had been received or which had geographical features apparently favorable for Culicoides infestations. An outdoors entomological survey using human landing catches was undertaken in areas reporting a pest problem. 


\begin{abstract}
Results. Culicoides infestations were confirmed as a serious nuisance problem in the rural areas of nine counties located in the western foothills of the Eastern Range of the Colombian Andes. Although available epidemiological records were fragmented, it was established that in six counties $11.4 \%$ of the dermatitis cases (total=2,472 cases) reported between 2003 and 2005 were attributed to the Culicoides bites. The entomological survey identified Culicoides pachymerus as the dominant species, $99.3 \%$ of 3,389 caught females. Biting rates in the most intensely affected areas reach a geometric mean of 52 females/person per 5 minutes. Multivariate analysis indicated that abundance of $C$. pachymerus had a negative relationship with altitude. Conclusions. Based on its dominance and high biting rates, $C$. pachymerus is probably the species responsible for the high nuisance levels caused by Culicoides bites and the associated dermatological pathology, within the study area.
\end{abstract}

Key words: Ceratopogonidae, dermatitis, public health, Andean ecosystem.

Los insectos del género Culicoides, pequeños dípteros hematófagos, de distribución cosmopolita, son importantes en salud pública por la molestia que ocasionan sus picaduras, en especial, cuando se presentan con gran abundancia y por su papel como transmisores de diversos agentes (virus, protozoos y nemátodos) que causan enfermedades a los humanos y a los animales $(1,2)$. Además, dependiendo del grado de sensibilidad de las personas a los componentes de la saliva de estos insectos, las picaduras pueden causar reacciones inmediatas o tardías que van desde dermatitis alérgicas, pápulas y pústulas -producto de sobreinfección por rascado-, hasta reacciones más graves como eccemas, descamación y cicatrices con alteración de la pigmentación de la piel (2-4).

La enfermedad más importante transmitida por Culicoides a humanos en el neotrópico es el virus Oropouche (Bunyavirus), cuyo vector principal es C. paraensis (Goeldi, 1905) (5-7), el cual se distribuye en la cuenca amazónica de Brasil y Perú, y en Panamá, Trinidad y Tobago (6). La sintomatología de la enfermedad es similar a la del dengue clásico con una duración de una semana, aproximadamente y, generalmente, no es fatal (8). Se estima que sólo en Brasil se han infectado cerca de 500.000 personas desde 1960 y se han presentado 27 epidemias (5).

Los Culicoides también pueden transmitir a los humanos nemátodos del género Mansonella,

\footnotetext{
Correspondencia:

Erika Santamaría, Grupo de Entomología, Instituto Nacional de Salud, Bogotá, D.C., Colombia. Telefax: (051) 2200923 esantamaria@ins.gov.co
}

Recibido: 28/02/08; aceptado:06/08/08 agentes causales de las denominadas mansonelosis, enfermedades de amplia distribución y cuadro clínico variable, pero sin efectos patológicos significativos. Entre los nemátodos Mansonella del Nuevo Mundo se destaca $M$. ozzardi, que se distribuye en la cuenca amazónica, Centroamérica y partes de Colombia, Ecuador, Bolivia y Argentina (1,9). C. furens (Poey, 1851) y $C$. phebotomus (Willistoni, 1896) se consideran como los vectores principales en Haití y Trinidad, respectivamente, y $C$. barbosai Wirth y Blanton, 1956 y C. paraensis, como algunos de los vectores secundarios (1). En Colombia no se ha incriminado ninguna especie de Culicoides aunque, basados en el desarrollo de microfilarias en insectos alimentados sobre voluntarios infectados, se sospecha de $C$. insinuatus Ortiz y León, 1955 como uno de los posibles vectores de $M$. ozzardi en el departamento del Amazonas (10).

En los animales la enfermedad más notable, de distribución mundial, transmitida por los Culicoides es la lengua azul, causada por un Orbivirus que afecta al ganado bovino y ovino. En la región del Caribe los vectores probables son $C$. filarifer Hoffman, 1939, C. insignis Lutz, 1913 y C. pusillus Lutz, 1913 (1). Se han presentado epidemias en varios países como Grecia, Turquía, Israel, España y Portugal (1). En Colombia, la abundancia de $C$. insignis se ha asociado con la transmisión del virus de la lengua azul encontrado en ganado bovino en el departamento de Antioquia (11).

En Colombia, la importancia en salud de los Culicoides se centra en la molestia causada por sus picaduras a humanos en una variedad de climas y regiones, la cual se ha reportado desde 
tiempos de la conquista española. En escritos que datan de 1570 se menciona que los Culicoides, conocidos como "jejenes", causaban una gran molestia por sus picaduras en el valle del Magdalena, el Cabo de la Vela y en el Orinoco (Valderrama R. Artropodosis en Colombia. Una visión histórica. Memorias, XXVI Congreso de la Sociedad Colombiana de Entomología, 1998). Más recientemente se han reportado como plagas a C. puracensis Wirth y Lee, 1967 en el páramo de Puracé (12) y a C. pachymerus Lutz, 1914 en las tierras cálidas del piedemonte y valle del Magdalena medio (13, datos sin publicar, Grupo de Entomología, Instituto Nacional de Salud). En relación con el conocimiento de la biología y ecología de los Culicoides, se ha publicado un catálogo parcial en el que se reportan, por lo menos, 88 especies (14) y un estudio intensivo en el departamento del Valle del Cauca, en donde se determinaron las especies antropofílicas, los hábitats más favorables y los criaderos para varias especies de Culicoides (15).

Desde la década de los 70 del siglo pasado, los habitantes de varios municipios del occidente del departamento de Boyacá, ubicados en el piedemonte del valle del Magdalena medio, han manifestado a las autoridades de salud su malestar por las constantes picaduras de los Culicoides que llegan, incluso, a causar lesiones dermatológicas complejas. Sin embargo, hasta el momento, no se ha realizado ningún estudio epidemiológico ni entomológico detallado que permita identificar aspectos básicos para abordar el control de esta problemática, tales como el área afectada, la prevalencia de las lesiones dermatológicas, la población en riesgo, las especies de Culicoides implicadas y los sitios y tiempo de contacto humano-Culicoides. Sólo se ha llevado a cabo un estudio entomológico previo en el municipio de Otanche, en donde se hicieron algunas observaciones sobre la actividad diaria de $C$. pachymerus, dentro de una evaluación preliminar de varios insecticidas para el control de Culicoides adultos con resultados cuestionables debido a falta de detalle en la descripción de la metodología empleada (13).

En el presente trabajo se muestran los resultados de un estudio exploratorio, realizado con el fin de identificar las áreas afectadas por la molestia sanitaria causada por las picaduras de Culicoides a humanos en el departamento de Boyacá, incluyendo la determinación de las especies involucradas y su abundancia.

\section{Materiales y métodos}

\section{Recolección de información sobre molestia sanitaria y problemas dermatológicos causados por Culicoides a nivel municipal}

Con el fin de tener una aproximación inicial a la problemática causada por los Culicoides en el departamento de Boyacá, en una reunión de octubre de 2005 se solicitó a los médicos de los centros de salud y técnicos de saneamiento de los municipios aparentemente afectados que presentaran en exposiciones orales, la información de los últimos 10 años sobre el número y procedencia de casos dermatológicos posiblemente asociados con picaduras de Culicoides, los tratamientos médicos y tradicionales, y los métodos de control de los Culicoides. Se invitaron a participar en la reunión a 22 municipios: 12 con reportes previos de molestia sanitaria causada por Culicoides, localizados en el piedemonte del flanco occidental de la Cordillera Oriental en la cuenca media del río Magdalena (datos sin publicar de la Secretaría de Salud Departamental de Boyacá) y 10 municipios ubicados en el flanco oriental de la cordillera, con condiciones geográficas similares a los primeros (piedemonte con altitudes alrededor de $1.000 \mathrm{msnm}$ o menor) (figura 1), pero sin reportes de molestia sanitaria.

\section{Muestreo entomológico}

El muestreo se realizó entre diciembre de 2005 y febrero de 2006 en el área rural de diez municipios que, en la reunión inicial para el diagnóstico de la problemática, confirmaron tener molestia sanitaria causada por los Culicoides. En cada municipio se seleccionaron entre dos y cinco veredas teniendo en cuenta las quejas de la comunidad por picadura de Culicoides y la presencia de casos clínicos de dermatitis causados, probablemente, por las picaduras de estos insectos. En cada vereda se muestrearon entre uno y tres sitios afuera de las viviendas. El intradomicilio no fue 
muestreado porque en este hábitat las abundancias de Culicoides son aparentemente bajas (13).

Los muestreos se hicieron con atrayente humano debido a que el principal interés del estudio era identificar las especies de Culicoides que causaban molestia por sus picaduras a humanos, y este método es la forma directa de determinar las especies antropofílicas. Para el muestreo, una persona voluntaria expuso una mano y antebrazo, mientras que otra persona (técnico de saneamiento) recolectó, con la ayuda de un pincel humedecido en alcohol, los Culicoides tan pronto como se posaban sobre la piel expuesta.

Las capturas se hicieron entre las $14: 00$ y las 17:30, periodo de mayor actividad de Culicoides en el área de estudio, según observaciones preliminares (13). La duración del tiempo promedio de muestreo fue variable según la abundancia de Culicoides: 10 minutos para sitios de alta abundancia ( $>40$ picaduras en 5 minutos) y 30 minutos para sitios con baja abundancia $(<40$ picaduras en 5 minutos). Lo anterior se hizo como medida de protección de las personas en sitios con posible alta abundancia de Culicoides. Los muestreos preliminares llevados a cabo por los autores, indicaban que en algunos sitios se presentaban tasas de picadura mayores a 50 hembras/persona en 5 minutos.

Para cada sitio de muestreo se registró la altitud aproximada, las distancias a la vivienda y a la corriente de agua (río o quebrada) más cercana y el tipo de hábitat (con base en las formas de vida de las plantas dominantes) dentro de un radio de $20 \mathrm{~m}$. Además, se estableció que los muestreos se llevarían a cabo en ausencia de lluvia para evitar el efecto negativo que puede tener esta variable sobre la actividad de los Culicoides en sitios abiertos y sin sombrío (16).

Los especímenes recolectados se preservaron en alcohol al $70 \%$ y, luego, se contaron e identificaron por especie en el laboratorio, teniendo en cuenta el patrón de pigmentación de las alas y las descripciones originales de varias especies de Culicoides (3,17-19). Las especies identificadas fueron confirmadas por Gustavo Spinelli del Museo de La Plata, Universidad de La Plata, Argentina. Se depositaron montajes permanentes de especímenes de las especies recolectadas en la colección entomológica del Grupo de Entomología del Instituto Nacional de Salud.

\section{Análisis de la información}

La información relacionada con el muestreo entomológico fue digitada en Epilnfo 3.3.2 desde donde se exportó a los programas Excel y Stata 7 para el análisis estadístico de la abundancia (tasa de picadura) de las especies de Culicoides más representativas del muestreo. La tasa de picadura se presenta como promedios geométricos del número de hembras/persona en 5 minutos con sus respectivos intervalos de confianza del $95 \%$. El ajuste de presentar la tasa de picadura estimada para un tiempo de 5 minutos fue necesario para hacer comparables los diferentes tiempos de muestreo.

Mediante el análisis multivariado se probó si seis variables explicativas: altitud, distancias a la vivienda y a la corriente de agua más cercana y los porcentajes de cobertura de los tres tipos de hábitat (bosque, rastrojo y herbáceas, principalmente pastizales) más comunes alrededor del sitio de muestreo (20 m de radio), podían explicar la abundancia de Culicoides (20). Estas variables se probaron como variables categóricas con los siguientes intervalos: a) altitud, cinco intervalos: 200-450, 451-700, 701-950, 951-1.200, 1.201$1.450 \mathrm{msnm}$; b) distancias a la vivienda y fuentes de agua más cercanas, cinco intervalos: 0-10, 11 50, 51-100, 101-200 y >200 m; y c) porcentaje de tipos de hábitat, seis clases: 0, 1-19, 20-39, 40$59,60-79$ y $80-100 \%$.

El análisis multivariado se realizó sobre los datos de abundancia de Culicoides transformados a $\sqrt{x}$ para lograr su normalización. En el análisis, primero se generó un modelo máximo que incluía las seis variables explicativas. Luego, el modelo se simplificó por eliminación secuencial de las variables menos significativas usando la prueba F. Finalmente, se obtuvo un modelo mínimo adecuado donde todas las variables fueron significativas $(p<0,05)$. Las abundancias de Culicoides por municipio se compararon mediante análisis univariado. 


\section{Consideraciones éticas}

El método de captura de los Culicoides con atrayente humano fue aprobado por el Comité de Ética del Instituto Nacional de Salud.

\section{Resultados}

\section{Descripción y delimitación del área afectada}

Sólo representantes de las autoridades de salud de 14 municipios, de los 22 municipios a los que se solicitó información sobre la molestia causada por Culicoides, asistieron a la reunión para exponer su problemática. Once de estos municipios se localizan sobre el flanco occidental de la Cordillera Oriental, mientras que los restantes tres municipios se encuentran sobre el flanco oriental de la misma cordillera (figura 1). Los ocho municipios que no enviaron representantes no lo hicieron porque sus autoridades de salud consideraron que los Culicoides no causaban una molestia significativa. La mayoría de estos municipios (7 de 8) se localizan sobre el flanco oriental de la Cordillera Oriental (figura 1).

Se consideró que un municipio estaba afectado por la problemática cuando sus representantes reportaron más del $30 \%$ de veredas con molestias por picadura de Culicoides o cuando había reportes de casos clínicos por las picaduras de estos insectos. Basados en los anteriores criterios, se puede decir que la problemática causada por los Culicoides en el departamento de Boyacá se concentra en el flanco occidental de la Cordillera Oriental, sobre la cuenca del río Minero, que incluye nueve municipios: Maripí (67\% de veredas afectadas), Muzo (58\%), San Pablo de Borbur (52\%), Tununguá (43\%), Otanche (40\%), La Victoria (36\%), Quípama (34\%), Coper
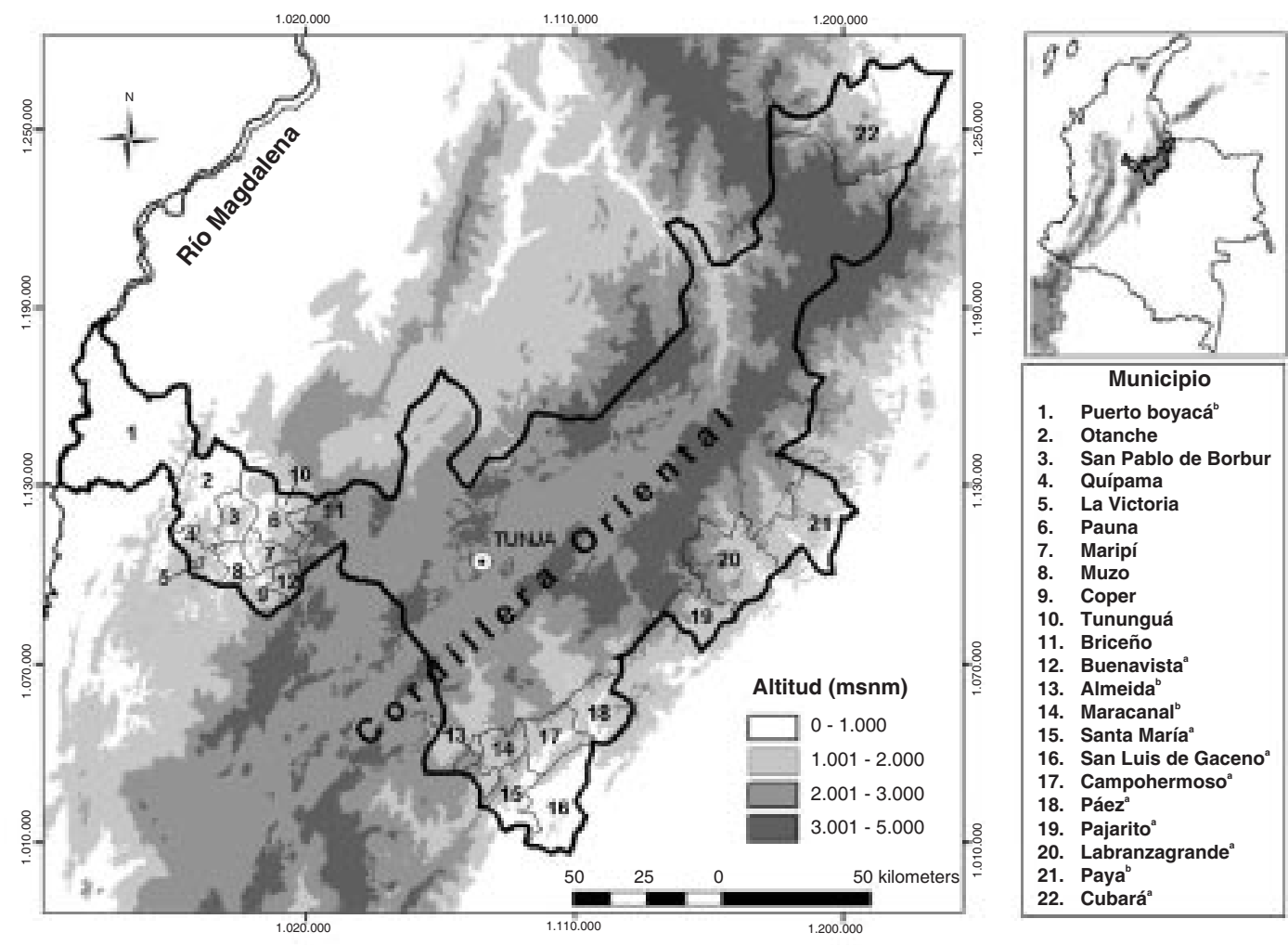

Figura 1. Municipios del departamento de Boyacá en donde se indagó sobre la molestia sanitaria causada por Culicoides y se hizo muestreo entomológico. En algunos municipios no se realizó el muestreo ( ${ }^{a}$ ocho que no asistieron a la reunión, y ${ }^{\mathrm{b}}$ tres que sí asistieron) porque las autoridades de salud consideraron que los Culicoides no eran una molestia. 
y Pauna (ambos municipios sólo con reporte de casos de dermatitis) (figura 1).

La molestia se localizó principalmente en el área rural, y afectó una población aproximada de 15.000 personas. Sin embargo, también se reportó molestia leve en la zona urbana de dos municipios, Muzo y San Pablo de Borbur. El área afectada corresponde principalmente a la zona de vida de Holdridge conocida como bosque húmedo tropical (bh-T) que se caracteriza por tener una precipitación anual entre 2.000 y $4.000 \mathrm{~mm}$, una temperatura media mayor de $24^{\circ} \mathrm{C}$ y una altitud entre 0 y 1.000 msnm. También, se encuentran presentes el bosque muy húmedo premontano (bmh-PM), el bosque húmedo premontano (bhPM) y el bosque seco tropical (bs-T).

Casuística de las manifestaciones clínicas, tratamientos y molestia sanitaria causadas por las picaduras de Culicoides

La información de reporte de casos de dermatitis causados por picadura de Culicoides en los municipios estudiados es incompleta, con un historial muy corto, menor de tres años, y probablemente con un alto subregistro. A pesar de lo anterior, esta información puede servir de punto de referencia para comparación con estudios futuros.

Sólo seis municipios (Otanche, Maripí, Macanal, Quípama, Muzo y Coper) informaron tener registros de casos de dermatitis, posiblemente causada por las picaduras de Culicoides, pero sólo para el periodo 2003 a 2005. En estos municipios se reportaron 2.472 casos, de los cuales, el 11,4\% (Quípama: 106 casos, Muzo: 99 casos y Coper: 77 casos) se atribuyó directamente a las picaduras de Culicoides. Cabe resaltar el alto número de casos de dermatitis reportados en 2005 y que correspondieron exclusivamente al municipio de Otanche $(n=1.694$ casos, cerca del $50 \%$ de la población rural del municipio) los cuales fueron detectados por búsqueda activa, en varias brigadas de salud desarrolladas durante ese año. Infortunadamente no hay un estimativo sobre la proporción de estos casos que pudo haber sido causada por picaduras de Culicoides. Los demás municipios manifestaron no contar con datos epidemiológicos sobre esta problemática, aunque se estima que, por lo menos, en un municipio (Pauna) la incidencia es alta.

La percepción de los médicos es que la comunidad parece estar acostumbrada a la dermatitis producida por las frecuentes y continuas picaduras de los Culicoides y que las personas sólo consultan cuando hay infecciones secundarias serias ocasionadas por el rascado o por condiciones higiénicas precarias. Entre los principales síntomas que los médicos han observado en personas afectadas por las picaduras de Culicoides están: edema, pápulas, eritema, prurito, hipertermia, urticaria, dermatosis, piel escamosa y queratosis. Las autoridades de salud de la mayoría de los municipios coincidieron en que la molestia por Culicoides tiene un gran impacto en la comunidad ya que, además de los problemas dermatológicos mencionados, las altas tasas de picadura de estos insectos interfieren en el desarrollo de las actividades diarias de los habitantes, y producen un deterioro de la calidad de vida.

Entre los tratamientos médicos para tratar los efectos de las picaduras se prescriben antipruriginosos locales, antihistamínicos, analgésicos y, en caso de sobreinfección, antibióticos tópicos y sistémicos. Existen pacientes que por presentar reacciones alérgicas graves requieren tratamientos más prolongados y con seguimiento. En cuanto a tratamientos tradicionales, se mencionó el uso tópico de limón con sal, alcohol, aguas de hierbas y emplastos de tabaco.

\section{Conocimiento de los Culicoides y su control}

De acuerdo con la información suministrada por los representantes de los municipios, los Culicoides son conocidos por la población con el nombre de "moscacilla", con excepción de Puerto Boyacá en donde los llaman "jejenes". Las administraciones municipales no aplican medidas dirigidas a controlar los Culicoides. Sin embargo, los pobladores usan hogueras en el peridomicilio y fumigan con insecticidas domésticos (Raid®, Baygón®) y derivados del petróleo (ACPM, querosene y gasolina). Además, tratan de reducir el contacto directo con los insectos mediante el uso de toldillos y la aplicación en la piel de 
repelentes (jabón Nopikex®), aceites (de cocina y Johnson®) y vaselina.

\section{Diagnóstico entomológico}

Se recolectaron 3.389 Culicoides en 76 muestras en 19,9 horas de muestreo, afuera de las viviendas de diez municipios. Todos los especímenes fueron hembras, distribuidas en cinco especies. C. pachymerus (figura 2) fue la especie dominante con $99,3 \%$ del total. Las otras cuatro especies $(0,3 \%)$ fueron: cuatro especímenes de $C$. debilipalpis Lutz, 1913, un espécimen de $C$. paraensis (Goeldi, 1905), tres especímenes de C. mirsae Ortiz, 1953 y un espécimen de C. leoni Barbasa, 1952. El restante 0,4\% correspondió a ejemplares incompletos que no se pudieron identificar hasta especie.

Los datos de abundancia de $C$. pachymerus presentaron una distribución agregada con un índice de dispersión $\left(I=s^{2} / \bar{x}\right)$ de 13,8. Por vereda, la distribución agregada de los datos de abundancia fue evidente (figura 3 ), con seis veredas en donde se concentraron las tasas de picadura más altas de esta especie (promedios geométricos entre 33 y 58 hembras/persona en 5 minutos): Santa Rosa, Ancamay, Centro, San Isidro, Chizo Cuepar y Chanares.

A nivel municipal, el patrón de agregación también se mantuvo (cuadro 1). La mayor tasa de picadura de $C$. pachymerus se encontró en San Pablo de Borbur, promedios geométricos de 52 hembras/ persona en 5 minutos, seguido por Tununguá, 29 hembras/persona en 5 minutos y Coper, 21 hembras/persona en 5 minutos. Los municipios con las menores tasas de picadura fueron Briceño, 2,6 hembras/persona en 5 minutos y La Victoria, 0,9 hembras/persona en 5 minutos. El análisis univariado mostró que estas diferencias en tasas de picadura entre municipios fueron muy significativas $\left(\mathrm{F}_{(9)(66)}=16,87, p<0,001\right)$ con la tasa de picadura de C. pachymerus en San Pablo de Borbur significativamente mayor que en cualquiera de los otros nueve municipios.

El cuadro 1 muestra las características del hábitat en $20 \mathrm{~m}$ de radio de los sitios de muestreo para cada uno de los municipios estudiados junto con la abundancia de $C$. pachymerus por municipio. A pesar de que a primera vista no se aprecian diferencias claras que puedan ayudar a explicar la variación en abundancia entre municipios, algunas tendencias se hacen aparentes al comparar los municipios con las tasas de picadura extremas.

El municipio con la mayor tasa de picadura de $C$. pachymerus (San Pablo de Borbur) presentó la menor altitud (mediana: $435 \mathrm{msnm}$ ) y distancias cortas a la vivienda (mediana: $30 \mathrm{~m}$ ) y a la corriente de agua más cercanas (mediana: $70 \mathrm{~m}$ ). En relación con el tipo de hábitat, en este municipio dominaron los bosques (50\%) y los pastizales (45\%). Por el contrario, el municipio con la menor tasa de picadura de C. pachymerus (La Victoria) presentó la mayor altitud (1.400 msnm), la mayor distancia a la vivienda más cercana $(300 \mathrm{~m})$ y

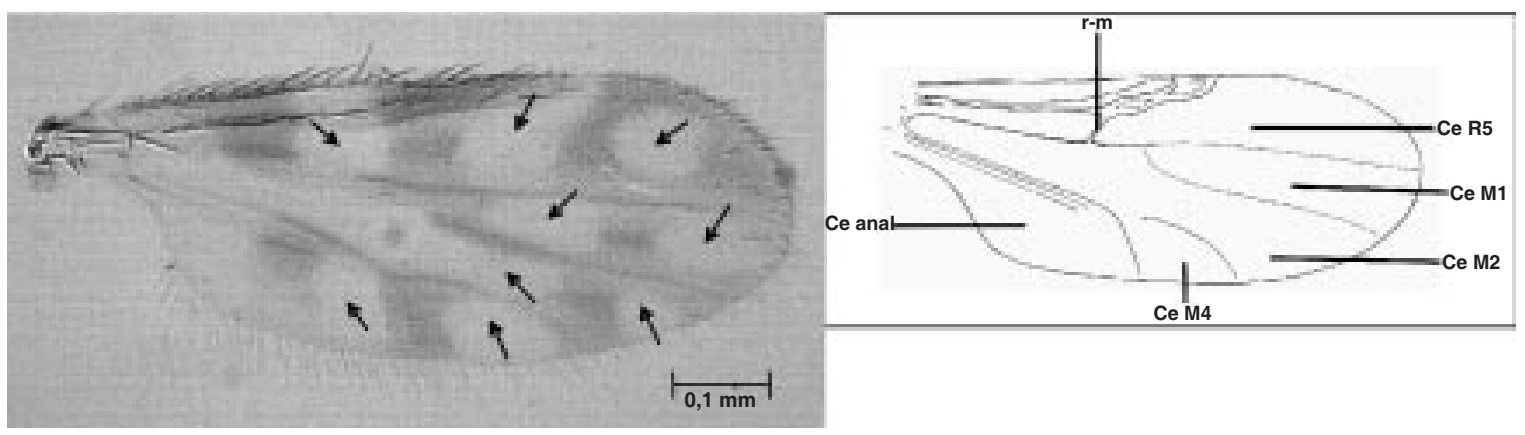

Figura 2. Ala de la hembra de Culicoides pachymerus que muestra su patrón de pigmentación (manchas claras señaladas con flechas). Distribución de las manchas: una sobre la vena r-m, dos en la celda R5 (Ce R5), dos en ambas Ce M1 y Ce M2, y una en ambas Ce M4 y Ce anal. El recuadro muestra la localización de las venas y celdas mencionadas. 


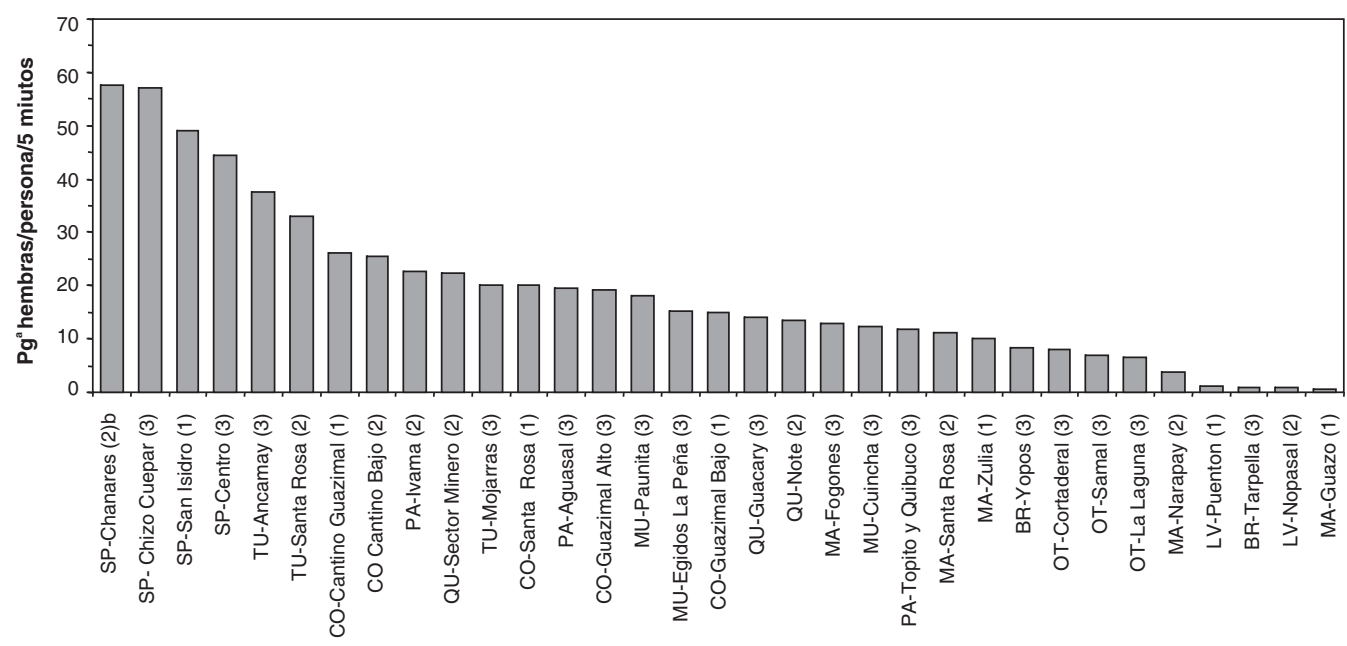

aaromedio geométrico; 'Tamaño de la muestra

Figura 3. Abundancia de Culicoides pachymerus afuera de las viviendas por vereda. El municipio al que pertenece cada vereda se indica mediante dos letras mayúsculas que anteceden al nombre: BR: Briceño, CO: Coper, LV: La Victoria, MA: Maripí, MU: Muzo, OT: Otanche, PA: Pauna, QU: Quípama, SP: San Pablo de Borbur, TU: Tununguá.

una distancia a la corriente de agua más cercana relativamente grande $(300 \mathrm{~m})$.

El análisis multivariado mostró a la altitud como la única variable explicativa de la abundancia de C. pachymerus con una asociación negativa (cuadro 2, figura 4). En el menor intervalo de altitud, 200 a $450 \mathrm{msnm}$, se presentó la tasa de picadura más alta de este insecto (promedios geométricos de 39 hembras/persona en 5 minutos) y en el mayor intervalo, 1.201-1.450 msnm, la tasa de picadura más baja (promedios geométricos de 0,9 hembras/persona en 5 minutos). Esta variable explicó el $36,5 \%\left(r^{2}=0,365\right)$ de la variación en la abundancia de $C$. pachymerus.

En dos municipios (Quípama y Coper), la comunidad reportó a las viviendas como sitios de alta actividad de picadura de los Culicoides, por lo que se realizaron cuatro muestreos adicionales en este hábitat. Específicamente, se muestreó el área abierta construida adyacente a los dormitorios: corredor, patio o la entrada de la vivienda. La mayor tasa de picadura de $C$. pachymerus, 51 hembras/persona en 5 minutos, se presentó en una vivienda del Sector Minero (Quípama). En las demás viviendas la tasa de picadura fue relativamente baja: 2,7 y 4,5 hembras/persona en 5 minutos en dos viviendas de la vereda Resguardo (Coper) y 7,8 hembras/ persona en 5 minutos en una vivienda de la vereda Note (Quípama).

\section{Discusión}

Los resultados del muestreo entomológico mostraron que $C$. pachymerus es prácticamente la única especie antropofílica, 99,3\% de los especímenes capturados, en los diez municipios muestreados. Esta amplia dominancia concuerda con un estudio previo realizado en el municipio de Otanche en el que $C$. pachymerus representó el $98,8 \%$ de las capturas, aparentemente también con atrayente humano (13). La amplia dominancia de $C$. pachymerus en el presente estudio se considera representativa del área muestreada. A pesar de que el tiempo de muestreo fue corto, tres meses, no parece que haya efectos estacionales que modifiquen la dominancia de $C$. pachymerus. Los muestreos posteriores al presente estudio realizados en varias épocas han confirmado la dominancia de C. pachymerus a lo largo del año (datos sin publicar, Grupo de Entomología, Instituto Nacional de Salud). 
Cuadro 1. Características del hábitat (20 m de radio) en los sitios muestreados por municipio y abundancia de Culicoides pachymerus afuera de las viviendas

\begin{tabular}{|c|c|c|c|c|c|c|c|c|c|c|}
\hline \multirow[b]{2}{*}{ Variable } & \multicolumn{9}{|c|}{ Municipio } & \multirow[b]{2}{*}{ La Victoria } \\
\hline & $\begin{array}{l}\text { San Pablo } \\
\text { de Borbur }\end{array}$ & Tununguá & Coper & Pauna & Quípama & Muzo & Otanche & Maripí & Briceño & \\
\hline Altitud (msnm) & $\begin{array}{c}435 \\
(408-450)^{\mathrm{e}}\end{array}$ & $\begin{array}{c}850 \\
(828-893)\end{array}$ & $\begin{array}{c}775 \\
(663-963)\end{array}$ & $\begin{array}{c}600 \\
(500-700)\end{array}$ & $\begin{array}{c}700 \\
(700-900)\end{array}$ & $\begin{array}{c}890 \\
(860-915)\end{array}$ & $\begin{array}{c}804 \\
(783-841)\end{array}$ & $\begin{array}{c}800 \\
(650-875)\end{array}$ & $\begin{array}{c}650 \\
(500-838)\end{array}$ & $\begin{array}{c}1400 \\
(1400-1400)\end{array}$ \\
\hline $\begin{array}{l}\text { Distancia a la vivienda } \\
\text { más cercana }(\mathrm{m})\end{array}$ & $\begin{array}{c}30 \\
(21-75)\end{array}$ & $\begin{array}{c}45 \\
(5-500)\end{array}$ & $\begin{array}{c}160 \\
(6-875)\end{array}$ & $\begin{array}{c}140 \\
(16-200)\end{array}$ & $\begin{array}{c}100 \\
(10-100)\end{array}$ & $\begin{array}{c}5 \\
(4-20)\end{array}$ & $\begin{array}{c}50 \\
(15-70)\end{array}$ & $\begin{array}{c}10 \\
(9-30)\end{array}$ & $\begin{array}{c}14 \\
(5-125)\end{array}$ & $\begin{array}{c}300 \\
(90-1000)\end{array}$ \\
\hline $\begin{array}{l}\text { Distancia a la corriente } \\
\text { de agua más cercana }(\mathrm{m})\end{array}$ & $\begin{array}{c}70 \\
(17-2500)\end{array}$ & $\begin{array}{c}500 \\
(136-1375)\end{array}$ & $\begin{array}{c}225 \\
(75-900)\end{array}$ & $\begin{array}{c}900 \\
(575-1000)\end{array}$ & $\begin{array}{c}50 \\
(30-200)\end{array}$ & $\begin{array}{c}40 \\
(2-200)\end{array}$ & $\begin{array}{c}95 \\
(70-500)\end{array}$ & $\begin{array}{c}300 \\
(150-1500)\end{array}$ & $\begin{array}{c}400 \\
(230-500)\end{array}$ & $\begin{array}{c}300 \\
(30-500)\end{array}$ \\
\hline \multicolumn{11}{|l|}{ Tipo de hábitat (20 m de radio) } \\
\hline $\begin{array}{l}\text { bosque } \%^{\mathrm{a}} \\
\text { altura }^{\mathrm{b}}(\mathrm{m})\end{array}$ & $\begin{array}{c}50 \\
(30-50) \\
30\end{array}$ & $\begin{array}{c}18 \\
(8-28) \\
15\end{array}$ & $\begin{array}{c}13 \\
(1-55) \\
30\end{array}$ & $\begin{array}{c}5 \\
(0-51) \\
15\end{array}$ & $\begin{array}{c}50 \\
(10-70) \\
15\end{array}$ & $\begin{array}{c}30 \\
(25-50) \\
15\end{array}$ & $\begin{array}{c}70 \\
(15-100) \\
7\end{array}$ & $\begin{array}{c}0 \\
(0-25) \\
9\end{array}$ & $\begin{array}{c}5 \\
(0-25) \\
7^{*}\end{array}$ & $\begin{array}{c}17 \\
(0-40) \\
14\end{array}$ \\
\hline $\begin{array}{l}\text { rastrojo } \% \\
\text { altura }(\mathrm{m})\end{array}$ & $\begin{array}{c}0 \\
(0-0) \\
3\end{array}$ & $\begin{array}{c}0 \\
(0-0) \\
2\end{array}$ & & $\begin{array}{c}20 \\
(10-30) \\
2\end{array}$ & $\begin{array}{c}0 \\
(0-0) \\
N D^{f}\end{array}$ & & $\begin{array}{c}0 \\
(0-30) \\
1\end{array}$ & $\begin{array}{c}0 \\
(0-10) \\
3\end{array}$ & $\begin{array}{c}10 \\
(0-33) \\
2,5\end{array}$ & $\begin{array}{c}50 \\
(0-70) \\
3^{*}\end{array}$ \\
\hline $\begin{array}{r}\text { herbáceo \% } \\
\text { (principalmente pastizales) } \\
\text { altura }(\mathrm{m})\end{array}$ & $\begin{array}{c}45 \\
(15-50) \\
0,5\end{array}$ & $\begin{array}{c}38 \\
(10-78) \\
0,5\end{array}$ & $\begin{array}{c}45 \\
(8-78) \\
0,5\end{array}$ & $\begin{array}{c}30 \\
(10-58) \\
0,3\end{array}$ & $\begin{array}{c}10 \\
(0-30) \\
1\end{array}$ & $\begin{array}{c}20 \\
(10-50) \\
0,05\end{array}$ & $\begin{array}{c}0 \\
(0-15) \\
0,08\end{array}$ & $\begin{array}{c}80 \\
(70-88) \\
0,5\end{array}$ & $\begin{array}{c}30 \\
(0-60) \\
0,5\end{array}$ & $\begin{array}{c}20 \\
(17-30) \\
1\end{array}$ \\
\hline $\begin{array}{r}\text { cultivos \% } \\
\text { (café, caña, árboles frutales) } \\
\text { altura }(\mathrm{m})\end{array}$ & $\begin{array}{c}0 \\
(0-0) \\
0,8\end{array}$ & $\begin{array}{c}0 \\
(0-0) \\
3\end{array}$ & $\begin{array}{c}5 \\
(0-30) \\
13\end{array}$ & $\begin{array}{c}0 \\
(0-0) \\
N D\end{array}$ & $\begin{array}{c}0 \\
(0-0) \\
N D\end{array}$ & & & $\begin{array}{c}0 \\
(0-5) \\
3,5\end{array}$ & $\begin{array}{c}10 \\
(0-43) \\
2,5\end{array}$ & \\
\hline terreno sin vegetación % & $\begin{array}{c}0 \\
(0-18)\end{array}$ & $\begin{array}{c}0 \\
(0-8)\end{array}$ & & $\begin{array}{c}5 \\
(0-34)\end{array}$ & $\begin{array}{c}0 \\
(0-70)\end{array}$ & & $\begin{array}{c}0 \\
(0-0)\end{array}$ & & $\begin{array}{c}25 \\
(0-35)\end{array}$ & \\
\hline carretera \% & & & & $\begin{array}{c}0 \\
(0-0)\end{array}$ & & $\begin{array}{c}0 \\
(0-20)\end{array}$ & & $\begin{array}{c}0 \\
(0-0)\end{array}$ & & \\
\hline $\begin{array}{r}\text { corriente de agua } \% \\
\text { (río o quebrada) }\end{array}$ & $\begin{array}{c}0 \\
(0-5)\end{array}$ & $\begin{array}{c}0 \\
(0-45)\end{array}$ & $\begin{array}{c}0 \\
(0-8)\end{array}$ & & $\begin{array}{c}10 \\
(0-30)\end{array}$ & $\begin{array}{c}0 \\
(0-50)\end{array}$ & & $\begin{array}{c}0 \\
(0-0)\end{array}$ & & $\begin{array}{c}30 \\
(0-30)\end{array}$ \\
\hline vegetación en ribera ${ }^{\mathrm{c}, \mathrm{d}}$ & 50 he, $50 \mathrm{sv}$ & $100 \mathrm{sv}$ & 50 ar, $50 \mathrm{he}$ & & $75 \mathrm{ar}, 25 \mathrm{sv}$ & $33 \mathrm{ar}, 67 \mathrm{sv}$ & & $100 \mathrm{he}^{*}$ & & $100 \mathrm{sv}$ \\
\hline $\begin{array}{l}\mathrm{PG}^{\mathrm{g}} \text { C. pachymerus } \\
\text { hembras/persona/5 } \mathrm{min} \\
\text { IC } 95 \%{ }^{\mathrm{h}} \\
\mathrm{n}^{1}\end{array}$ & $\begin{array}{c}52 \\
(44-61) \\
9\end{array}$ & $\begin{array}{c}29 \\
(21-40) \\
8\end{array}$ & $\begin{array}{c}21 \\
(17-25) \\
8\end{array}$ & $\begin{array}{c}17 \\
(7-40) \\
8\end{array}$ & $\begin{array}{c}16 \\
(10-25) \\
7\end{array}$ & $\begin{array}{c}15 \\
(10-22) \\
9\end{array}$ & $\begin{array}{c}7,1 \\
(4-12) \\
9\end{array}$ & $\begin{array}{c}6,6 \\
(2,9-15) \\
9\end{array}$ & $\begin{array}{c}2,6 \\
(0,5-14) \\
6\end{array}$ & $\begin{array}{c}0,9 \\
(0,4-1,7) \\
3\end{array}$ \\
\hline
\end{tabular}

a Mediana del porcentaje de un tipo de hábitat específico; ${ }^{\mathrm{b}}$ Altura mediana; ${ }^{\circ}$ Porcentaje de sitios con muestreos positivos; ${ }^{\mathrm{d}}$ ar: árboles, he: herbáceas, sv: sin vegetación; ${ }^{\mathrm{e} C u a r t i l e s}$

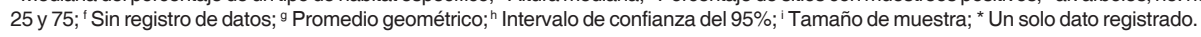

Cuadro 2. Variables, dentro de un radio de $20 \mathrm{~m}$ alrededor de los sitios de muestreo, que determinan la abundancia de C. pachymerus afuera de las viviendas, identificadas mediante análisis multivariado.

\begin{tabular}{|c|c|c|c|c|c|}
\hline Variable & & coeficiente & $z$ & $p$ & $\begin{array}{c}\text { Intervalo de confianza } \\
\text { del } 95 \%\end{array}$ \\
\hline \multirow[t]{6}{*}{ Altitud (msnm) } & $200-450^{a}$ & & & & \\
\hline & $451-700$ & $-2,30$ & $-3,71$ & $<0,001$ & $-3,51 \quad-1,08$ \\
\hline & $701-950$ & $-2,71$ & $-4,71$ & $<0,001$ & $-3,84 \quad-1,58$ \\
\hline & $951-1200$ & $-1,91$ & $-1,53$ & 0,125 & $-4,35 \quad 0,53$ \\
\hline & $1201-1450$ & $-5,56$ & $-5,24$ & $<0,001$ & $-7,63 \quad-3,48$ \\
\hline & Intersección & 6,49 & 12,76 & $<0,001$ & $5,49 \quad 7,49$ \\
\hline
\end{tabular}

\footnotetext{
${ }^{a}$ Categoría de referencia para las comparaciones
} 


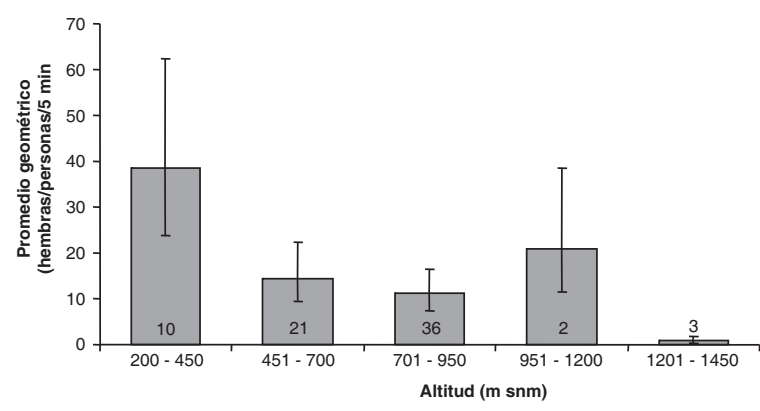

Figura 4. Abundancia afuera de las viviendas de Culicoides pachymerus en relación con la altitud. El número en la base de las barras indica el tamaño de muestra. Las barras de error corresponden al intervalo de confianza del $95 \%$.

C. pachymerus tiene una distribución amplia: Guatemala, Panamá, Ecuador, Venezuela, Colombia y Brasil. A pesar de lo anterior, sólo se ha reportado como causante de molestia sanitaria significativa en Colombia, en donde ha sido reportada en localidades ubicadas sobre el valle del río Magdalena en los departamentos de Huila (municipio de Neiva) y Tolima (Honda, Armero, Mariquita, Armero, El Guamo y Carmen de Apicalá) (14; datos sin publicar, Grupo de Entomología, Instituto Nacional de Salud). En la mayoría de localidades se tiene información de que esta especie es molestia sanitaria (datos sin publicar, Grupo de Entomología, Instituto Nacional de Salud).

En relación con las otras especies de Culicoides reportadas en el presente estudio, $C$. leoni, $C$. paraensis, C. mirsae y C. debilipalpis son nuevos registros para el departamento de Boyacá. Teniendo en cuenta las limitaciones de tiempo (muestreo sólo durante la estación seca) y métodos de muestreo (únicamente atrayente humano) se considera que la riqueza de especies de Culicoides es mucho mayor a las cinco especies registradas en el presente trabajo.

En principio, las tasas de picadura del presente estudio no se pueden comparar directamente con las de otros estudios por variaciones en los tiempos de muestreo y en la forma de medir las tasas, promedios geométricos en este estudio y promedios aritméticos en previos estudios. Sin embargo, se considera que las tasas de picadura de $C$. pachymerus por municipio fueron altas en el área de estudio comparadas con las de otras investigaciones, teniendo en cuenta que los promedios geométricos fueron casi iguales a los aritméticos. La tasa de picadura estimada por hora fluctuó desde un promedio geométrico de 11 hembras/persona por hora $(0,9$ hembras/persona en 5 minutos), en el municipio de Briceño, hasta 624 hembras/persona por hora (52 hembras/persona en 5 minutos) en San Pablo de Borbur.

En el estudio de Villarreal (13), en seis veredas del municipio de Otanche, las tasas de picadura fluctuaron tan sólo entre 17 y 232 insectos por persona por hora. Las altas tasas de picadura de C. pachymerus en los municipios estudiados del departamento de Boyacá confirman la molestia sanitaria a la que está expuesta la población. Las tasas de picadura más altas han sido reportadas para $C$. furens en Jamaica y $C$. impunctatus en Alaska, con alrededor de 1.000 insectos por persona por hora $(21,22)$. Un caso excepcional es el de C. tristriatulus, en Alaska, especie con la que se ha registrado una tasa de picadura de 300 insectos por minuto (por ejemplo, 18.000 insectos por hora) (23). Las altas tasas de picadura han hecho que varias especies de Culicoides (es decir, $C$. furens, $C$. impunctatus o $C$. ornatus) sean consideradas plagas para el hombre y sus animales domésticos, llegando en ocasiones a frenar el desarrollo económico y turístico en Puerto Rico, Panamá, Estados Unidos y Australia (24-27).

En relación con la información epidemiológica, aunque se presentaron reportes de consultas médicas por afecciones dermatológicas probablemente asociadas con las picaduras de los Culicoides, el bajo porcentaje $(11,4 \%)$ de casos confirmados y la falta de información detallada sobre sexo y edad, impiden hacer una descripción detallada de esta problemática. Por lo anterior, es necesario realizar un estudio epidemiológico, que incluya aspectos entomológicos, en el área de estudio en el que se determinen las características y la magnitud real de la problemática. Sin embargo, fue clara la importancia de la molestia sanitaria, evidente en el alto porcentaje de veredas reportadas como afectadas por municipio (36\% a $64 \%$ ). De ninguna otra región de Colombia se han publicado estudios 
sobre reacciones dermatológicas en humanos causadas por las picaduras de Culicoides.

En Brasil, en la ciudad de Salvador (estado de Bahía) en el término de un año, se registraron en un hospital 211 casos con graves reacciones en la piel causadas por las picaduras de $C$. paraensis, con una aparente mayor incidencia en las mujeres, atribuida a su forma de vestir que permite una mayor exposición de las piernas a las picaduras de insectos, y en la población joven (28). En Nigeria, se estudió la percepción de un grupo de personas respecto a la molestia ocasionada por $C$. fulvithorax, y se encontró que el $86,5 \%$ (739/854) había sufrido reacciones cutáneas, como inflamación y prurito, a causa de las picaduras de esta especie (29).

Aunque a nivel municipal no hubo una evidente asociación positiva aparente entre la abundancia de $C$. pachymerus registrada en el muestreo entomológico y el reporte de las autoridades de salud del porcentaje de veredas afectadas por municipio por las picaduras de estos insectos (ver descripción y delimitación del área de estudio y el cuadro 1), sí se observó que la mayoría de municipios con las menores tasas de picadura ( $\leq 2,6$ hembras/persona en 5 minutos, para Briceño y La Victoria) no reportaron o reportaron los menores porcentajes ( $0 \%$ y $36 \%$, respectivamente) de veredas afectadas por molestia sanitaria causada por los Culicoides, y que el municipio con la tasa de picadura más alta (San Pablo de Borbur: 52 hembras/persona en 5 minutos) reportó uno de los mayores porcentajes (52\%) de veredas afectadas.

Las altas dominancia y tasa de picadura de $C$. pachymerus, más su aparente mayor abundancia en la mayoría de municipios más afectados por la molestia sanitaria y problemas dermatológicos, indican que esta especie es la responsable de la problemática en mención en el departamento de Boyacá.

En cuanto al riesgo potencial de sufrir molestia sanitaria y problemas dermatológicos causados por la picadura de $C$. pachymerus al que pueden estar expuestos los habitantes del área de estudio, teniendo en cuenta sólo las tasas de picadura de C. pachymerus por municipio, puede decirse que los municipios con mayor riesgo (tasas de picadura >20 hembras/persona en 5 minutos) son San Pablo de Borbur, Tununguá y Coper. Con un riesgo medio (tasas de picadura entre 6 y 20 hembras/ persona en 5 minutos) están Pauna, Quípama, Muzo, Otanche y Maripí. Los municipios con el más bajo riesgo (tasas de picadura $<6$ hembras/ persona en 5 minutos) son La Victoria y Briceño.

En cuanto al efecto de algunas variables del sitio de muestreo sobre la tasa de picadura de $C$. pachymerus, el análisis multivariado mostró que la altitud fue la única relacionada con la tasa de picadura, con una asociación negativa y mayor abundancia en el rango de 200-400 msnm. Esto coincide con el conocimiento empírico de las autoridades de salud de algunos municipios, para las cuales la molestia se concentraba en veredas con altitudes por debajo de los $1.000 \mathrm{msnm}$. Esta variable puede ser considerada como un factor de riesgo potencial. Es de anotar que la gran mayoría de los 123 municipios que conforman el departamento de Boyacá están localizados sobre altitudes mayores a los 2.000 msnm (figura 1) y en ellos no se tiene antecedentes de que los Culicoides causen problemas a la población humana.

Aunque la distancia a la vivienda y a la corriente de agua más cercanas no se relacionaron estadísticamente con la tasa de picadura de $C$. pachymerus, las mayores tasas de picadura de esta especie en los sitios cercanos a estos hábitats sugieren que el entorno cercano a las viviendas (peridomicilio) y las corrientes de agua son los más favorables para la actividad de los adultos y probablemente para el desarrollo de sus estadios inmaduros. El peridomicilio es uno de los sitios reconocidos como de mayor actividad de picadura para algunas especies de Culicoides como, por ejemplo, C. paraensis (16). De otra parte, es bien conocido que las corrientes de agua son uno de los diversos hábitats húmedos en donde los Culicoides pueden criarse $(5,14)$.

Estudios recientes han asociado mediante análisis multivariado la abundancia o la presencia de Culicoides, capturados con trampas de luz, con variables bióticas y abióticas. En un estudio a lo largo de Italia se encontró que las seis 
variables probadas (altitud, pendiente del terreno, temperatura, porcentaje de cobertura con bosque, índice de aridez y el índice normalizado de vegetación diferencial) están asociados con la abundancia de C. imicola y con el complejo Obsoletus. C. imicola se asocia con altitudes bajas y hábitats con vegetación dispersa y expuesta al sol, mientras que el complejo Obsoletus se asocia con áreas de mayor altitud y con hábitats más sombreados (30). En Australia, en un estudio del movimiento de poblaciones de C. brevitarsis desde una zona costera hacia el interior del continente, se encontró que la barrera de altitud representada por una cordillera limita los movimientos de la especie en mención (31).

En conclusión, los resultados entomológicos y epidemiológicos del presente estudio indican que C. pachymerus es causante de la molestia sanitaria y al menos de buena parte de los problemas dermatológicos por picaduras de insectos en el departamento de Boyacá. La molestia se concentra por debajo de los $1.000 \mathrm{msnm}$ en el piedemonte de la Cordillera Oriental que rodea el valle medio del río Magdalena.

\section{Agradecimientos}

A las autoridades de salud que recopilaron la información sobre la problemática causada por Culicoides en sus municipios y a los técnicos de saneamiento ambiental que colaboraron con los muestreos entomológicos.

\section{Conflictos de intereses}

Los autores declaran que no existe conflicto de intereses.

\section{Financiación}

Colciencias (proyecto código: 2104-04-16499), Instituto Nacional de Salud y Secretaría de Salud de Boyacá.

\section{Referencias}

1. Mullen GR. Biting midges (Ceratopogonidae). En: Mullen G, Durden L, editors. Medical and veterinary entomology. San Diego: Academic Press; 2002. p. 163-83.

2. Kettle DS. Ceratopogonidae (Biting midges). En: Kettle DS, editor. Medical and veterinary entomology. Second edition. Cambridge: CAB Internacional; 1995. p. 152-76.
3. Forattini OP. Culicoides da Regiao Neotropical (Diptera: Ceratopogonidae). Arq Fac Hig Saude Publica Univ Sao Paulo. 1957;11:161-526.

4. Sherlock IA, Guitton N. Dermatozoonosis by Culicoides'bite (Diptera: Ceratopogonidae) in Salvador, state of Bahia, Brazil. IV. A clinical study. Mem Inst Oswaldo Cruz. 1965;63:27-37.

5. Borkent A. The biting midges, the Ceratopogonidae (Diptera). En: Marquardt WC, editor. Biology of disease vectors. Second edition. Burlington MA: Elsevier Academic Press; 2005. p. 113-26.

6. Tesh RB. The emerging epidemiology of Venezuelan hemorrhagic fever and Oropouche fever in tropical South America. Ann NY Acad Sci. 1994;740:129-37.

7. Linley JR, Hoch AL, Pinheiro FP. Biting midges (Diptera: Ceratopogonidae) and human health. J Med Entomol. 1983;20:347-64.

8. Leduc JW, Hoch AL, Pinheiro FP, Travassos Da Rosa AP. Epidemic Oropouche virus disease in northern Brazil. Bull Pan Am Health Organ. 1981;15:97-103.

9. Undiano C. Importance and present-day concepts of pathogenicity of Mansonella infections. Rev Fac Ciencias Med Córdoba. 1966;24:183-9.

10. Tidwell MA, Tidwell MA. Development of Manzonella ozzardi in Simulium amazonicum, S. argentiscutum and Culicoides insinuatus from Amazonas, Colombia. Am J Trop Med Hyg. 1982;31:1137-41.

11. Homan EJ, Taylor WP, De Ruiz L, Yuill TM. Bluetongue virus and epizootic haemorrhagic disease of deer virus serotypes in northern Colombian cattle. J Hyg (Lond). 1985;95:165-72.

12. Rodriguez MA, Wirth WW. A new species of manbiting Culicoides from the high Andes of Colombia (Diptera: Ceratopogonidae). Florida Ent. 1986;69:311-4.

13. Villarreal LI. Estrategia de control de Culicoides sp., en el departamento de Boyacá. Boletín Epidemiológico de Boyacá. Tunja: Secretaría de Salud de Boyacá. 1998. p. $105-10$.

14. Barreto P. Catálogo de los Culicoides (Diptera: Ceratopogonidae) de Colombia. Colombia Med. 1986;17:140-50.

15. Browne JE. Light-trap population studies of the Culicoides from three life zones in Colombia with notes on biting habits and larval habitats (Diptera: Ceratopogonidae) (thesis). New Orleans: Tulane University; 1978. p. 134.

16. Hoch AL, Roberts DR, Pinheiro FP. Host-seeking behavior and seasonal abundance of Culicoides paraensis (Diptera: Psychodidae) in Brazil. J Am Mosq Control Assoc. 1990;6:110-4.

17. Wirth WW, Dyce AL, Spinelli GR. An atlas of wing photographs, with a summary of the numerical charac- 
ters of the neotropical species of Culicoides (Diptera: Ceratopogonidae). Contrib Am Ent Inst. 1988;25:1-72.

18. Spinelli G, Wirth W. Clave para la identificación de las especies del género Culicoides presentes al sur de la Cuenca Amazónica, nuevas citas y notas sinonímicas (Diptera: Ceratopogonidae). Rev Soc Entomol Argentina. 1985;44:49-75.

19. Wirth WW, Blanton FS. Biting midges of the genus Culicoides from Panama (Diptera: Heleidae). Proceedings of the United States National Museum. 1959;109:237-482.

20. Crawley MJ. GLIM for ecologist. Oxford: Blackwell Scientific Publications: 1993. p. 380.

21. Kettle DS, Linley JR. The biting habits of Jamaican sandflies. Preferences for individuals, limbs and site positions. Part I Introduction and Culicoides barbosai Wirth y Blanton. Jamaica: Ministry of Health; 1960.

22. Jenkins DW. Ecological observations on the blackflies and punkies of Central Alaska. Mosq News. 1948;8: 148-54.

23. Sailer RI, Marks EP, Lienk S. Notes on Culicoides in Alaska (Diptera, Heleidae). Mosq News. 1956;16:270-8.

24. Fox I. Notes on Puerto Rican biting midges or Culicoides (Diptera, Ceratopogonidae). Bull Brooklyn Entomol Soc. 1949;44:29-34.

25. Carpenter SJ. Studies of Culicoides in the Panama Canal Zone (Diptera, Heleidae). Mosq News. $1951 ; 2: 202-8$
26. Blanton FS, Wirth WW. The sand flies (Culicoides) of Florida (Diptera: Ceratopogonidae). Arthropods of Florida 1979;10:1-204.

27. Whelan P. Biting midges or "sandflies" in the Northern Territory. The Northern Territory Disease Control Bulletin. 2003;10:1-10.

28. Sherlock IA, Guitton N. Dermatozoonosis by Culicoides'bite (Diptera: Ceratopogonidae) in Salvador, State of Bahia, Brazil. III. Epidemiological aspects. Mem Ins Oswaldo Cruz. 1965;63:1-12.

29. Agbolade OM, Akinboye DO, Olateju TM, Ayanbiyi OA, Kuloyo OO, Fenuga 0O. Biting of anthropophilic Culicoides fulvithorax (Diptera: Ceratopogonidae), a vector of Mansonella perstans in Nigeria. Korean $\mathrm{J}$ Parasitol. 2006;44:67-72.

30. Conte A, Goffredo M, Ippoliti C, Meiswinkel R. Influence of biotic and abiotic factors on the distribution and abundance of Culicoides imicola and the Obsoletus complex in Italy. Vet Parasitol. 2007;150:333-44.

31. Bishop AL, Spohr LJ, Barchia IM. Effects of altitude, distance and waves of movement on the dispersal in Australia of the arbovirus vector, Culicoides brevitarsis Kieffer (Diptera: Ceratopogonidae). Preventive Vet Med. 2004;65:135-45. 